\title{
Pharmaceutical applications of 3D printing technology: current understanding and future perspectives
}

\author{
Byeong Ju Park ${ }^{1} \cdot$ Ho Jae Choi ${ }^{1}$. Sang Ji Moon ${ }^{1}$. Seong Jun Kim ${ }^{1} \cdot$ Rajiv Bajracharya ${ }^{1}$. Jeong Youn Min ${ }^{1}$. \\ Hyo-Kyung Han ${ }^{1}$
}

Received: 19 June 2018 / Accepted: 26 October 2018 / Published online: 29 October 2018

(c) The Author(s) 2018, corrected publication 2019

\begin{abstract}
Three-dimensional printing (3DP) technology allows the fabrication of 3D objects with various geometrics in a layer-bylayer process. Some advantages of 3DP methods over the conventional manufacturing processes include the customization of medicines with individually adjusted doses, the ability to fabricate the sophisticated and complex solid dosage forms, on-demand manufacturing, and cost-effectiveness. Furthermore, recent years have seen an increasing interest in applying 3DP technology to the pharmaceutical manufacturing of drug products and development of various drug delivery systems. However, although 3DP technology exhibits many potential medical and economic benefits, there are also some technical and regulatory challenges restricting the wide applications of 3DP technology to pharmaceutical products. Accordingly, continuous innovation and refinement in 3DP methods are needed to overcome the current limitations and facilitate patientspecific health care with on-demand tailored medications in the future. This review introduces some 3DP techniques suitable for pharmaceutical manufacturing and also their applications to the development of drug dosage forms, indicating the feasibility of this technology in regular commercial production.
\end{abstract}

Keywords 3D printing $\cdot$ Dosage forms $\cdot$ Personalized medicine $\cdot$ Pharmaco-printing $\cdot$ Polypill

$\begin{array}{ll}\text { Abbreviations } \\ \text { 3DP } & \text { Three-dimensional printing } \\ \text { CIJ } & \text { Continuous inkjet printing } \\ \text { CLIP } & \text { Continuous liquid interface production } \\ \text { DLP } & \text { Digital light projection } \\ \text { DOD } & \text { Drop-on-demand printing } \\ \text { EBM } & \text { Electron beam melting } \\ \text { ER } & \text { Extended release } \\ \text { FDM } & \text { Fused deposition modeling } \\ \text { IR } & \text { Immediate release } \\ \text { PAM } & \text { Pressure-assisted microsyringe } \\ \text { PAM2 } & \text { Piston-assisted microsyringe } \\ \text { SLA } & \text { Stereolithography } \\ \text { SLM } & \text { Selective laser melting } \\ \text { SLS } & \text { Selective laser sintering }\end{array}$

Hyo-Kyung Han

hkhan@dongguk.edu

1 College of Pharmacy, Dongguk University-Seoul, Dongguk-ro-32, Ilsan-Donggu, Goyang, South Korea

\section{Introduction}

Three-dimensional printing (3DP) is a method to produce $3 \mathrm{D}$ objects from digital models by fusing or depositing materials in successive layers, which allows the fabrication of objects with various geometrics in a layer-by-layer process. The process is also called additive manufacturing, rapid prototyping, or solid free-form fabrication (Goole and Amighi 2016). 3DP technology has been available since the late 1980s and has been used in engineering and various non-medical manufacturing areas, including automotive, aerospace, and consumer goods industries (Alhnan et al. 2016), however, rapid advances in 3DP methods and the emergence of versatile biocompatible materials facilitate the pharmaceutical applications of 3DP technology in recent years. Following the first medical applications of 3DP for the fabrication of custom prosthetics and dental implants, in the early 2000s, the 3DP technology has been since used to directly print medical devices having highly complex 3D architectures, and fabricate medical devices personalized to fit a patient's own anatomy (Maulvi et al. 2017). Furthermore, it has the potential to reduce the risk of failure at the later stages of new drug development process, as this 
technique can be deployed to fabricate more predictable drug screening platforms (Peng et al. 2017).

Due to its many inherent advantages over the conventional technologies, including the customization and personalization of medicines with individually adjusted doses, the ability to fabricate complex solid dosage forms with high accuracy and precision, on-demand manufacturing, and costeffectiveness, the application of 3DP methods to the pharmaceutical manufacturing of drug products gains a great attention in recent years. For example, various drug delivery systems have been developed using 3DP technology, such as oral controlled released systems, microchips, implants, pills, immediate release (IR) tablets, and multiphase release dosage forms. Given that personalized dosage forms are desirable to avoid unnecessary adverse effects, correct the dose regimen, and achieve personalized release profiles, the employment of rapidly evolving 3DP technologies also supports the fabrication of personalized drug delivery systems (Alhnan et al. 2016). In 2015, the FDA approved Spritam ${ }^{\circledR}$ (Levetiracetam), the first 3D-printed drug product, which opened a new chapter of pharmacoprinting in pharmaceutical manufacturing (Norman et al. 2017).

This review will introduce some 3DP technologies suitable for pharmaceutical manufacturing and also their applications to the development of dosage forms, indicating the feasibility of this technology in regular commercial production.

\section{DP technologies applicable to pharmaceutical developments}

Various 3DP methods have been developed by varying its energy source, material source, and other mechanical characters. Among them, the common 3DP technologies applicable for pharmaceutical areas are printing-based inkjet (IJ) systems, nozzle-based deposition systems, and laser-based writing systems (Murphy and Atala 2014), which can be further divided into several subtypes, depending on the materials and energy sources. A brief overview of the general characteristics of each 3DP technology is provided in the following sections.

\section{Printing-based inkjet (IJ) system}

IJ systems comprise two types of technologies, namely, continuous inkjet printing (CIJ) and drop-on-demand printing (DOD). CIJ technology creates a continuous stream of ink through an orifice of 50-80 $\mu \mathrm{m}$ diameter, by using a highpressure pump, whereas, DOD technology produces droplets of 10-50 $\mu \mathrm{m}$ with a volume of 1-70 pL (Konta et al. 2017). Both IJ systems possess a printer head and need to control the speed, size, and interval of drop formations and also fluid viscosity. The DOD system can use two types of printer head: a thermal head or a piezoelectric crystal (Goole and Amighi 2016). In thermal DOD, also called bubble jet printing, the ink is heated locally and forms bubbles that eject ink. In piezoelectric DOD, the rapid change in the shape of piezoelectric crystal results in a sudden volume change, producing an acoustic pulse sufficient for the ejection of ink (Gans-de et al. 2004). This piezoelectric DOD method can be used with a diverse range of liquids, while the thermal DOD method is limited to volatile liquids (Goole and Amighi 2016). In addition, given that (1) the thermal method reaches temperatures of up to $300^{\circ} \mathrm{C}$, which may cause the degradation of drugs, and (2) the piezoelectric DOD method can be operated at room temperature with less volatile and more biocompatible liquids, the piezoelectric DOD method may be more suitable for pharmaceutical applications.

The DOD technology can be further divided into two subtypes, termed drop-on-drop deposition and drop-on solid deposition (Goole and Amighi 2016). In drop-on-drop deposition, the printer head ejects the droplets onto each other to produce a solid layer, resulting in a high resolution 3D structure. This direct writing IJ-printing method is capable of fabricating microscopic drug delivery systems having diverse geometries, where droplet size is about $100 \mu \mathrm{m}$ in diameter and layer thicknesses are smaller than the droplet size maybe due to surface wetting, solvent evaporation, or shrinkage (Norman et al. 2017). The entire formulation in printable fluid should be suitable for jetting and rapid solidification (Norman et al. 2017). The physical properties of the printable fluid, such as viscosity and volatility, are also important to prevent the coffee ring effect, fluid leaking, and nozzle clogging (Goole and Amighi 2016). The optimum viscosity is reported to between 8 and $14 \mathrm{cps}$ (Sumerel et al. 2006). The physicochemical and the therapeutic properties of the incorporating drugs also affect the loading capacity and stability of the product (Goole and Amighi 2016).

Compared to drop-on-drop deposition, drop-on-solid deposition seems to be more applicable for the pharmacoprinting of a wide range of drugs, from chemical entities to biomolecules. Drop-on-solid deposition is also termed dropon-powder or drop-on-bed deposition, binder jetting, plaster printing, or powder bed 3DP, since it spreads the solid materials (powder) on the top of a platform and sprays the binder (a liquid ink) on the powders selectively (Goole and Amighi 2016; Gross et al. 2014). The platform is then lowered, and a new powder layer is spread again, repeating the process until a 3D structure is generated (Goole and Amighi 2016). The powder bed that is generally of $200 \mu \mathrm{m}$ in height and of which particle size ranges from 50 to $100 \mu \mathrm{m}$, is adhered to each other by the binder ink to create a 3D object (Gross et al. 2014). The layer thickness and spacing between each layer should be optimized for improved adhesion between layers. The reactivity of the powder bed with the binder ink and the topological characteristics of the powder are critical 
factors affecting the quality of the final products (Goole and Amighi 2016).

\section{Nozzle-based deposition systems}

Considering that the drawbacks of the most common printing-based IJ method are insufficient hardness, a rough surface, and low drug loadings (Dimitrov et al. 2006), nozzlebased deposition systems would be a suitable alternative to overcome those limitations. Instead of dropping the binder solution on a powder bed, nozzle-based deposition systems mix the solid components with the binder prior to 3D printing and directly deposit the mixture through a nozzle to create a 3D object (Vaezi et al. 2013). This method can be divided into two subtypes, named fused deposition modeling (FDM) and pressure-assisted microsyringes (PAM), according to the process with or without material melting, respectively.

FDM is one of the most commonly used 3DP techniques and has been well-studied in many fields, including pharmaceuticals, foods, and bioengineering (Alhijjaj et al. 2016). FDM, which is also called fused filament fabrication, defines a process in which a molten thermoplastic polymer filament is extruded through a high-temperature nozzle and deposited layer-by-layer with immediate solidification onto a build plate (Goyanes et al. 2015a). A simplistic illustration of FDM is provided in Fig. 1a. In pharmaceutical products, the active pharmaceutical ingredient (API) and thermoplastic polymer are mixed together via incubation in a certain solvent or melted together at an appropriate temperature before being extruded into filament. FDM is a low-cost manufacturing process and exhibits some advantages since it allows the production of highly complex drugs with difficult geometries and offers good mechanical strength, as well as options to modify the drug release profiles (Konta et al. 2017). However, there are also some drawbacks that limit its pharmaceutical applications, such as high operation temperatures, and few options regarding biodegradable thermoplastic polymers with good melt viscosity properties for extrusion.

PAM is another nozzle-based deposition system. In PAM, viscous and semi-liquid materials are extruded from a microsyringe (Goole and Amighi 2016). This syringe can move like an IJ printer head, and the semi-liquid material is released by compressed air. PAM technology allows producing microstructure of 5-10 $\mu \mathrm{m}$ or less (Vozzi et al. 2003). It is also able to create complex drug delivery systems and is advantageous over other methods since it can be operated in a continuous flow at room temperatures. However, the use of solvents may lead to safety and stability issues during the manufacturing and drying steps (Goole and Amighi 2016; Konta et al. 2017). Piston-assisted microsyringe (PAM2) is a rapid prototyping technology similar to PAM, but PAM2 use a stepper motor to release the printing materials rather than compressed air (Tirella et al. 2011).
Fig. 1 Graphical illustrations of 3DP processes. A FDM printer, B SLA printer, C DLP printer, D SLS printer
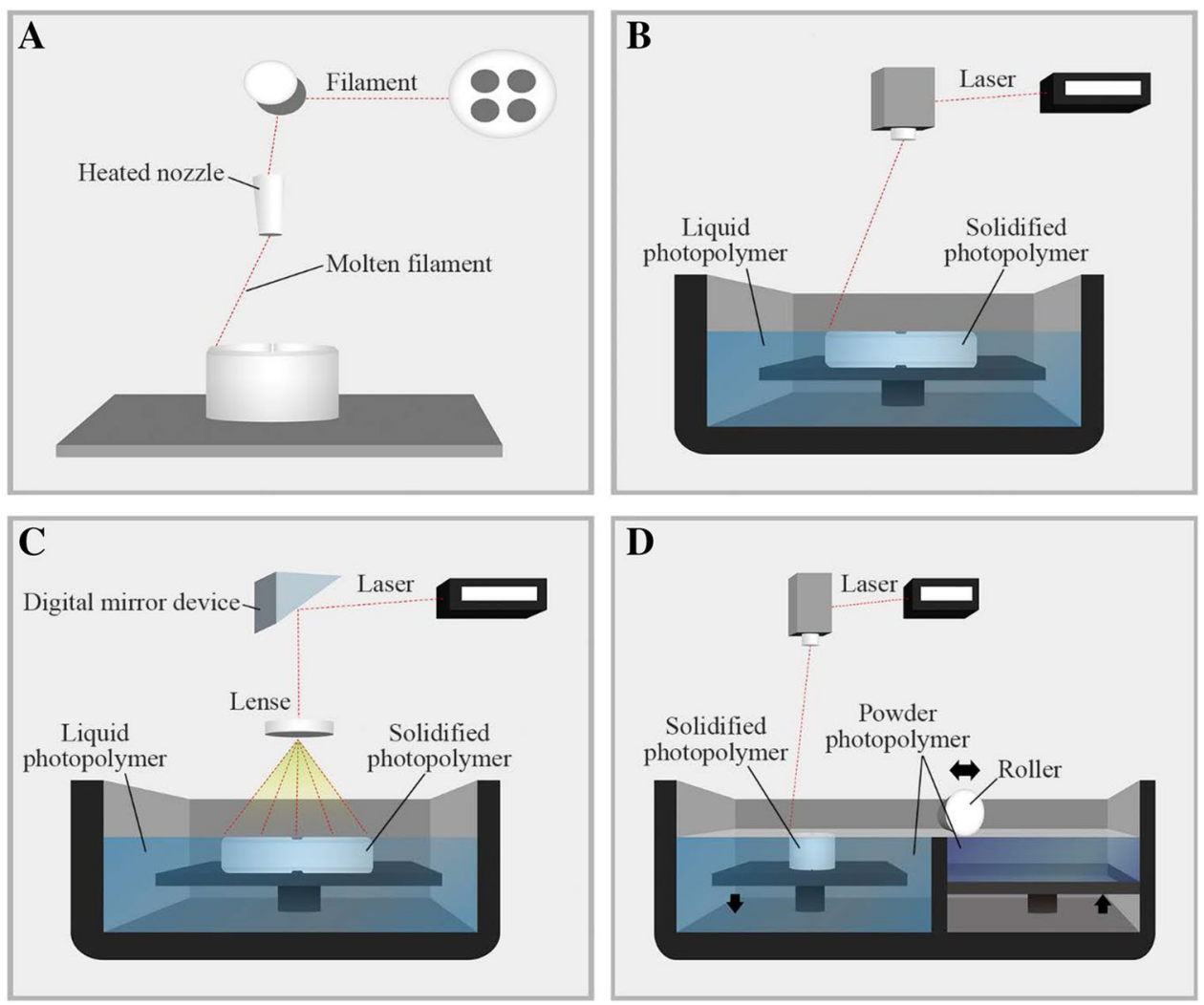


\section{Laser-based writing systems}

Stereolithography (SLA) is a laser-based printing method that was the first commercially available solid freeform fabrication technique. The fabrication of a 3D object by SLA is based on the controlled solidification of a liquid resin by photo-polymerization (Chia and $\mathrm{Wu} 2015$ ). A movable platform is located in the vessel filled with liquid photopolymer. First, the lifting platform starts near the surface of the liquid photopolymer, and after the proper laser is applied, the platform is lowered into a vessel to a depth equivalent to the thickness of the new polymerized layer (Fig. 1b). This process is repeated until a solid 3D product is obtained. SLA has high resolution which allows creating a complex structure, and it also minimizes the heating during the printing process, and thereby it is highly applicable to thermo-labile drugs (Konta et al. 2017). The choice of photopolymer is essential, as it should be a liquid that quickly solidifies upon illumination with ultraviolet (UV) light and, also, it must be approved for human use. Accordingly, the lack of Food and Drug Administration (FDA)-approved photosensitive polymers and the low drug loading significantly limit the pharmaceutical applications of SLA, although it is widely used in tissue engineering (Melchels et al. 2010). Digital light projection (DLP) is an emerging 3DP technique similar to SLA, which also uses liquid photopolymer resins and a laser beam to solidify and build an object (Kim et al. 2016). The difference between DLP and SLA is the use of a digital mirror device, allowing for the curing of a single layer at once, as illustrated in Fig. 1c. The simultaneous control of the millions of mirrors allows an entire layer to be cured at once, resulting in substantially reducing the layer production time (Gross et al. 2014). Accordingly, DLP offers a comparatively faster building and easy adjustment of the layer thickness.

Selective laser sintering (SLS) uses a high-power laser as an energy source to sinter a powder photopolymer (Fig. 1d). Once the laser selectively fuses the powder photopolymer, the platform supporting polymer lowers to refill with powder. High-strength, chemical resistance, and speed are advantages acquired by SLS technology. A similar technology to SLS is direct metal laser sintering (DMLS). However, while DMLS is applied to metal alloys, SLS is applied to a wide range of materials, including polymers, metals, and ceramics (Gross et al. 2014). Electron beam melting (EBM) and selective laser melting (SLM) are also similar to SLS. However, unlike sintering technology, both EBM and SLM completely melt metal powders during the layer-by-layer process. The SLM method uses energy from a laser beam to fuse the powder particles by heating it beyond the melting point while EBM uses a high-power electron beam in a vacuum. EBM can provide higher throughput and more uniform thermal field distribution than SLS, but its accuracy and surface quality are lower (Bikas et al. 2016). EBM and SLM are widely used in drug-loaded implants (Bezuidenhout et al. 2015).

In addition to the 3DP technologies described above, there are many other 3DP technologies available, including multi-jetting modeling, selective heat sintering, and laminated object manufacturing. Those 3DP technologies are not currently used for pharmaceutical manufacturing, but some of them have a high potential for pharmaceutical applications in the future. Therefore, a great advance in material sciences and the emergence of new adequate materials will facilitate more broad applications of various 3DP technologies.

\section{Application of 3DP technology to pharmaceutical dosage forms}

3DP technologies such as IJ, FDM, and SLS, are currently available for manufacturing adequate pharmaceutical dosage forms, as exemplified in Table 1. In this review, the pharmaceutical applications of 3DP technology are focused on the oral solid dosage forms and transdermal delivery systems that seem to be undergoing relatively greater progress and are more suitable for wide applications of 3DP.

\section{Tablets}

Oral dosage formulations are the most preferred form of pharmaceutical products. Tablets and capsules are typical examples of widely used solid oral dosage forms. Particularly, tablets have been extensively examined for the feasibility of 3DP technologies in pharmaceutical manufacturing. Generally, tablets produced by 3DP methods can be categorized into two groups: single API tablets and multiple API tablets. Selective examples of each category are described in the next two sections, respectively.

\section{Single API tablets}

Initially, 3DP technology was applied to fabricate simple immediate release (IR) tablets comprising a single API. In many studies, the FDM method was adopted for producing IR tablets, probably due to its simple fabricating procedures. Selective examples of single API IR tablets obtained by using the FDM method are reported in previous studies (Goyanes et al. 2016a; Okwuosa et al. 2016; Sadia et al. 2016). Not only low drug-loaded dosage forms but also high drug-loaded dosage forms can be prepared using 3DP technology. For example, a thermoplastic polyurethane-based dosage form loaded with $60 \%$ drug was successfully developed via the FDM method (Verstraete et al. 2018). Similarly, an IR tablet loaded with a very high dose 
Table 1 Selective examples of pharmaceutical dosage forms fabricated by 3DP

\begin{tabular}{|c|c|c|c|}
\hline Dosage form & 3DP method & API & References \\
\hline \multirow[t]{14}{*}{ Tablets } & \multirow[t]{5}{*}{ Inkjet system } & Acetaminophen & Yu et al. $(2007,2009)$ \\
\hline & & Chlorpheniramine maleate & Katstra et al. (2000) \\
\hline & & Chlorpheriramine maleate, diclofenac & Rowe et al. (2000) \\
\hline & & Levetiracetam & Jacob et al. (2014) \\
\hline & & Pseudoephedrine $\mathrm{HCl}$ & Wang et al. (2006) \\
\hline & Laser assisted system & 4-Aminosalicyclic acid, paracetamol & Wang et al. (2016) \\
\hline & \multirow[t]{8}{*}{ Fused deposition } & 5-Aminosalicylic acid, 4-aminosalicylic acid & Goyanes et al. (2015a) \\
\hline & & 5-Amino salicylic acid, theophylline, prednisolone & Sadia et al. (2016) \\
\hline & & $\begin{array}{l}\text { Aspirin, hydrochlorothiazide, atenolol, pravastatin sodium } \\
\text { and ramipril }\end{array}$ & Khaled et al. (2015a) \\
\hline & & Captopril, nifedipine and glipizide & Khaled et al. (2015b) \\
\hline & & Guaifenesin & Khaled et al. (2014) \\
\hline & & Paracetamol & Goyanes et al. (2015d) \\
\hline & & Prednisolone & Skowyra et al. (2015) \\
\hline & & Theophylline & Pietrzak et al. (2015) \\
\hline \multirow[t]{4}{*}{ Implant } & & 5-Fluorouracil & Yi et al. (2016) \\
\hline & & Isoniazid and rifampicin & Wu et al. (2009) \\
\hline & & Levofloxacin & Huang et al. (2007) \\
\hline & & Levofloxacin and tobramycin & Wu et al. (2016) \\
\hline \multirow[t]{4}{*}{ Microneedles } & & Dacarbazine & Lu et al. (2015) \\
\hline & & Diclofenac & Lim et al. (2017) \\
\hline & & Insulin & Pere et al. (2018) \\
\hline & & Rhodamine & Johnson et al. (2016) \\
\hline
\end{tabular}

of $80 \%$ paracetamol was prepared using an extrusion-based 3D printer (Khaled et al. 2018). In addition to IR tablets, 3DP is applicable to produce extended release (ER) tablets. Skowyra et al. (2015) examined the feasibility of the FDM method to fabricate ER tablets using prednisolone loadedpolyvinyl alcohol filaments, achieving the drug release up to $24 \mathrm{~h}$ (Skowyra et al. 2015). Another example of ER tablets prepared by the FDM method was reported by Alhijjaj et al. (2016), using polymer blends of polyethylene glycol, Tween 80 , and polyethylene oxide with either Eudragit ${ }^{\circledR}$ EPO or Soluplus ${ }^{\circledR}$.

When fabricating tablets by using 3DP techniques, the selection of 3DP materials and methods dramatically affects the physical properties of the obtained tablets, leading to the different drug release profiles. The ratios of the formulation components also influence the physical properties of tablets, resulting in altered drug release profiles. For example, Wang et al. (2006) have developed three near zero-order controlled-release pseudoephedrine hydrochloride dosage forms by using 3DP technology. The drug release rates were adjusted by varying the proportion of Kollidon ${ }^{\circledR} \mathrm{SR}$ and hydroxypropylmethyl cellulose (HPMC) while the fabrication parameters retained constant (Wang et al. 2006). These formulations also showed a good correlation between their in vitro dissolution profiles and clinical pharmacokinetic parameters. The doughnut-shaped multi-layered acetaminophen delivery devices were also developed by varying the amount of drug and release-retardant materials using 3DP technology, providing linear release profiles of a poorly water-soluble drug (Yu et al. 2009). In addition to immediate or extended drug release, 3DP technology is applicable for other types of modified release tablets. Using three different grades of hypromellose acetate succinate (grades LG, MG and $\mathrm{HG}$ ), enteric tablets were manufactured by the FDM method to enable manufacturing the delayed-release tablets without the need for an outer enteric coating (Goyanes et al. 2017). Furthermore, 3D extrusion-based printing has the potential to fabricate gastro-floating tablets (Li et al. 2018).

Other 3DP methods such as SLA and IJ method are also available for manufacturing tablets (Kyobula et al. 2017; Wang et al. 2016). For example, SLA was successful in fabricating ER tablets of 4-aminosalicylic acid or paracetamol, using polyethylene glycol diacrylate, diphenyl(2,4,6trimethylbenzoyl)phosphine oxide, and polyethylene glycol at various composition ratios, where the drug release profiles varied depending on the formulation compositions (Wang et al. 2016). Recently, Clark et al. (2017) developed a ropinirole hydrochloride tablet using the IJ method with UV photoinitiation and created the possibility for the translation of scalable, high-precision, and customized IJ-based 
additive manufacturing to the pharmaceutical sector (Clark et al. 2017). Kyobula et al. (2017) also showed that drugloaded solid dosage forms with complex geometries, such as honeycomb architecture, can be manufactured using hot melt 3D IJ printing and also indicated that variation in drug release profiles could be obtained in a controllable manner, by combining the geometrical capability of 3D printing with predictive computational approaches.

Taken together, IJ and FDM processes are currently the most promising approaches to the manufacturing of oral solid dosage forms. Particularly, FDM has shown a potential to prepare modified release dosage forms with complex geometries, but the number of polymer excipients is limited (Verstraete et al. 2018). Selected examples of polymers available for FDM are summarized in the Table 2. Although polymeric excipients modifying drug release properties are limited, the use of polymer blends may provide another option to overcome this issue. The interplay of the miscibility between excipients in the blends, the solubility of the materials in the dissolution media and the degree of fusion between the printed strips during the FDM process may affect the drug release rate from the matrix (Alhijjaj et al. 2016).

\section{Multiple API tablets}

To combine complex medication regimes into one, multiple APIs can be loaded in a single tablet, called a polypill. In recent studies, 3DP technology has been used to manufacture polypills showing controlled release profiles (Khaled et al. 2015a; Sun and Soh 2015). First, Khaled et al. (2015a) produced the polypill of captopril, nifedipine, and glipizide by using $3 \mathrm{D}$ extrusion-based printing, to treat patients with diabetes, suffering from hypertension. This polypill was composed of a captopril osmotic pump compartment, joining layer, and sustained release compartments of nifedipine and glipizide (Fig. 2a). After taking the pill, the joining layer was disintegrated quickly, thereby the polypill split into a captopril compartment and sustained release compartment. The captopril compartment showed zero-order drug release
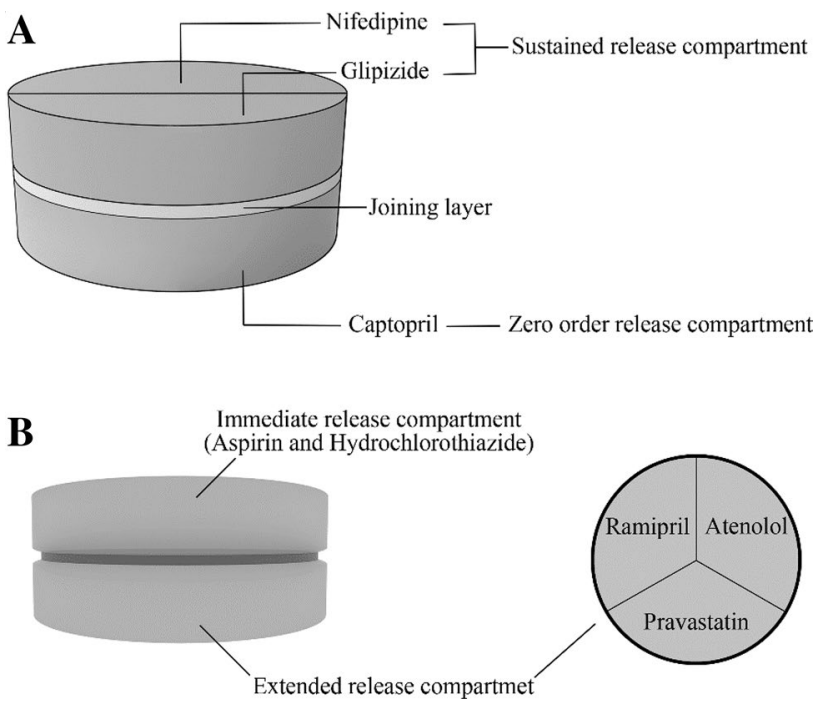

Fig. 2 Schematic illustrations of polypills containing multiple active ingredients (APIs). a Polypill with three APIs, b polypill with five APIs

based on the osmotic release of the drug through a controlled porosity shell while the sustained release compartments released the drugs (nifedipine and glipizide) via diffusion through gel layers (Khaled et al. 2015b). They also applied 3DP technology to fabricate a polypill containing five APIs (Khaled et al. 2015b). This polypill comprised two compartments showing independent controlled release profiles; one for sustained release and the other for immediate release (Fig. 2b). The sustained release compartment contained ramipril, atenolol, and pravastatin while the immediate release compartment contained aspirin and hydrochlorothiazide. In a 3DP extrusion system, the cellulose acetate shell is first extruded, and then the APIs (ramipril, atenolol, pravastatin) were mixed with HPMC. Rapid hydration of HPMC forms a gel-like state, leading to sustained drug release. In a next step, aspirin and hydrochlorothiazide were extruded to cover the top of the sustained release compartment, displaying immediate drug release due to the inclusion of a disintegrant (sodium starch glycolate). Through this drug
Table 2 Selective examples of modified-release polymers for FDM

\begin{tabular}{lll}
\hline Polymers & Model drug & References \\
\hline Cellulose (EC/HPC/HPMC)/Eudragit L100 & Acetaminophen & Zhang et al. (2017) \\
Ethylene vinyl acetate & Indomethacin & Genina et al. (2016) \\
Eudragit RL 100 & Deflazacort & Beck et al. (2017) \\
Hypromellose acetate succinate & Paracetamol & Goyanes et al. (2017) \\
Methacrylic polymers (Eudragit RL, RS and E)/HPC & Theophylline & Pietrzak et al. (2015) \\
Poly(ع-caprolactone) & Deflazacort & Beck et al. (2017) \\
Poly(L-lactic acid) & Nitrofurantoin & Sandler et al. (2014) \\
Polyvinyl alcohol & Fluorescein & Goyanes et al. (2014) \\
\hline
\end{tabular}

$H P C$ hydroxypropyl cellulose, $H P M C$ hydroxypropylmethyl cellulose, $E C$ ethyl cellulose 
combination in a tablet, patients who have various risk factors such as hypertension and dyslipidemia, can be treated simultaneously by just one tablet (Khaled et al. 2015a).

To use 3DP to control more complex release profiles, various shapes of drug carrier templates (or molds) are fabricated. Through complex templates, it is possible to create tablets that contain multiple components, to generate a multi-action releasing profile. In this way, APIs are not just released in zero- or first-order but more complicated release profiles can be acquired. Recently, Sun and Soh (2015) reported a 3DP method to fabricate customizable tablets which can achieve any desired release profile. The tablet is composed of three components, such as a surface-eroding polymer with the drug, surface-eroding polymer without the drug, and an impermeable polymer that forms a protective coating (Fig. 3). Particularly, the surface-eroding polymer containing the drug is fabricated with a specific shape, allowing the desired drug release profile. Varying the shape of the surface-eroding polymer carrying the drug leads to the different drug release profiles. Constant release, increasing release, decreasing release, and pulse release are acquired through designing the surface-eroding polymer with drug compartment. Complicated drug release, like pulse release, can be used for a drug that needs to be synchronized with the biological cycles of the patient. Decreasing release can be used in cases that require a relatively large dose of drug initially to act against a target rapidly, followed by gradually lower levels to prevent toxicity. As this method can modulate the drug release profiles through the shape of inner compartment, it seems promising to make customized drug tablets for complicated medications (Sun and Soh 2015).

Goyanes et al. (2015b) also reported the potential of 3DP to fabricate multiple-drug containing devices, with specialized design configurations and unique drug release characteristics. They used a multi-nozzle 3D printer to fabricate capsule-shaped solid devices loaded with acetaminophen and caffeine. The design structures included a multi-layer device, in which each layer contained a different drug, and a two-compartment device comprising a caplet embedded within a larger caplet (DuoCaplet), with each compartment containing a different drug. They demonstrated the unique drug release profiles, depending on the macrostructure of the devices. In multi-layer devices, the release of both drugs was simultaneous and independent of the drug solubility. In the DuoCaplet design, it was possible to achieve either immediate release or delayed release, by selecting the site of incorporation of the drug in the device and also the characteristics of the external layer (Goyanes et al. 2015b). This research group also developed modified release oral dosage forms (caplets) of budesonide using the FDM method combined with hot melt extrusion and fluid bed coating (Goyanes et al. 2015c). Wang et al. (2016) demonstrated the use of the SLA method to fabricate modified release tablets loaded with both
A

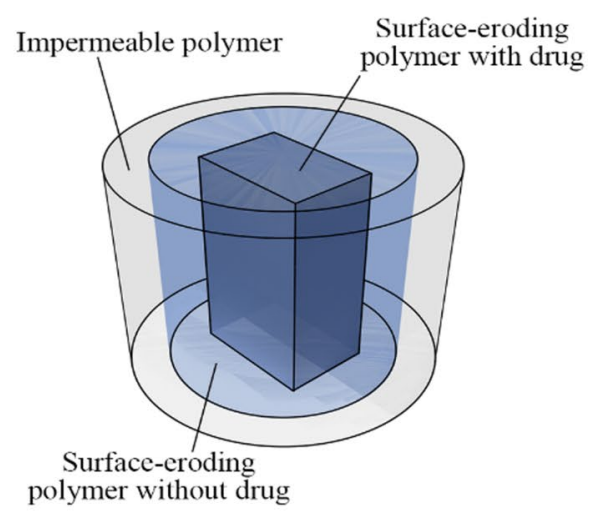

B

(a)
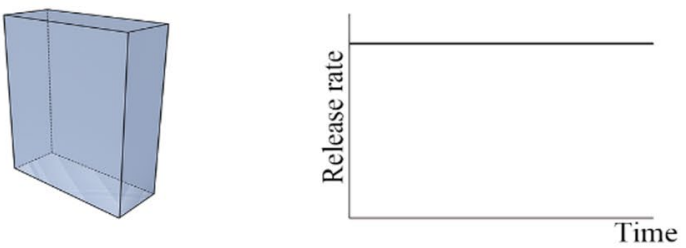

(b)
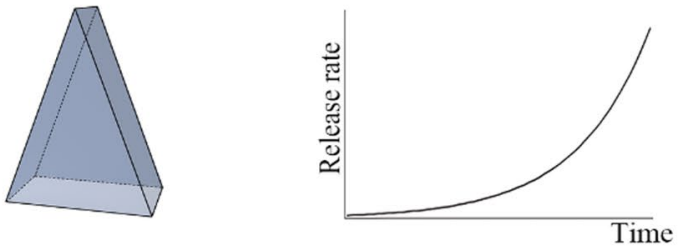

(c)
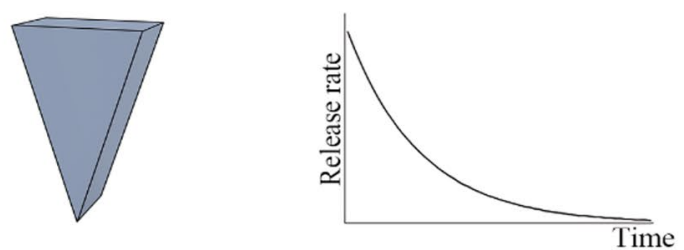

(d)
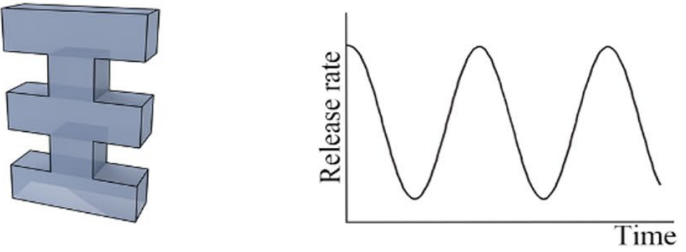

Fig. 3 Structural examples of release-controlled tablets. A Scheme of the release-controlled tablet, $\mathbf{B}$ examples of drug release profiles from each surface-eroding polymer in different shapes, representing constant drug release (a), increasing drug release (b), decreasing drug release $(\mathbf{c})$, and multiple-pulse drug release $(\mathbf{d})$, respectively

4-aminosalicylic acid (thermo-labile) and paracetamol (thermostable), suggesting that SLA reduces the drug degradation compared to FDM and offers an alternative way to produce tablets with thermo-sensitive drugs (Wang et al. 2016). 
In summary, 3DP technology has a high potential to manufacture various polypills containing multiple APIs in a tablet and also to achieve the complex and sophisticated drug release profiles. These polypills can help patients to rely on comparatively fewer pills, improve patient compliance, and allow personalized dosing regimens. Although there are still many limitations to apply 3DP to the pharmaceutical manufacturing process, 3DP would be an inexpensive and efficient way to produce the customized tablets.

\section{Implants}

An implant is a dosage form containing active drugs within a sustained release delivery matrix, providing the benefits to patients who need long-term treatment of drugs. While the traditional approach for implant development was mainly focused on extended and prolonged drug release, recent 3DP-based implants are designed to have complex micro- and macro-structures in a single device, for multi APIs loading and achieving more sophisticated drug release characteristics. For example, Huang et al. (2007) fabricated the implant of levofloxacin with predefined microstructure, exhibiting complex release profiles from a single implant. This implant displayed a bimodal profile, with pulsatile (day 5-25) and steady-state drug release (day 25-50), and then the pulse release began again on day 50 and continued up to day 80 .

Fabricating scaffolds for bone defect healing is one of the developing fields using 3DP. Particularly, in recent studies, multiple APIs were loaded inside the 3DP-based implants to acquire additional therapeutic effects. For bone defect healing, complex scaffolds were fabricated using 3DP, via combining calcium phosphate cement with vascular endothelial growth factor (VEGF)-loaded hydrogel strands (Ahlfeld et al. 2017). Wu et al. (2009) designed a multi-drug implant for bone tuberculosis treatment, using 3D printers. Isoniazid and rifampicin were incorporated into each layer alternatively in a specific sequence, forming a multi-layer concentric cylinder. Drugs were released sequentially from the outside layer to the center, creating a multi-drug therapeutic system. Due to their ideal pharmacological action and cytocompatibility, 3DP-based multi-drug implants could be a promising approach for the treatment of bone tuberculosis (Wu et al. 2009). Recently, Wu et al. (2016) designed a 3DP-based multi-drug implant for chronic osteomyelitis. Levofloxacin (LVFX) and tobramycin (TOB) were loaded into an implant as APIs, and the scaffolds were designed as multilayers where each layer's volume was $0.4 \mathrm{~cm}^{3}$ (Wu et al. 2016). The odd number layers were loaded with LVFX, and the even number layers were loaded with TOB. In this implant, each layer released API stepwise and exhibited a sustained drug release for 60 days to retain the ideal drug concentration, leading to the successful control of chronic osteomyelitis in rabbits (Wu et al. 2016).

A patch-like implant is a new type of implant developed by 3DP and focused on extended drug release for chronic diseases. For treatment of pancreatic cancer, Yi et al. (2016) applied 3DP to fabricate an implant designed appropriately for a specific tissue or organ (in this case, the pancreas) so that the implants could allow site-selective drug release. Poly-lactic-co-glycolic acid, polycaprolactone, and 5-fluorouracil were blended to produce the biodegradable implant patch with high drug loading efficiency (90\%). This patch was attached directly to the pancreas of an athymic mice model and showed significant efficacy, suggesting the utility of 3DP-based biodegradable implants for effective local delivery of anticancer drugs (Yi et al. 2016).

Owing to the high performance, controlled drug release for an extended duration, and effective local delivery, 3DPbased implants appear to be a promising dosage form for chronic diseases and expands the pharmaceutical applications of 3DP technology.

\section{Microneedles}

Microneedles are a class of transdermal drug delivery systems, which has arrays of micron-sized needles on the surface of a matrix to enhance the skin penetration of biologically active molecules. Notably, microneedles may be more effective to deliver macromolecules through the skin than traditional patches, due to its microstructure. Recent advances in high-resolution 3DP techniques fabricating small and tiny structures, accelerate the application of 3DP in manufacturing the microneedles. While traditional microfabrication techniques are limited to the microneedles with simple geometries, new 3DP technology enables to fabricate microneedles having more sophisticated and complex geometries. Some selected examples are described in the remaining paragraphs of this section.

Ochoa et al. (2015) developed a new fabrication process for polymeric microneedles of complex geometries, by coupling 3DP with hydrogel casting/shrinking techniques. This technique effectively enhanced the resolution limit of 3DP and fabricated sharp microneedles having tips with $9.6 \mu \mathrm{m}$ radius of curvature, which may be applicable for vaccine delivery. Continuous liquid interface production (CLIP) has also been developed using photoreactive resin and UV light to form 3D structures, like pyramids, pillars, or even more complicated structures (Johnson et al. 2016). In this method, CLIP microneedles were composed of trimethylolpropane triacrylate, polyacrylic acid, and photopolymerizable derivatives of polyethylene glycol and polycaprolactone, and the fabrication of microneedles was a mold-independent onestep process, requiring less than $10 \mathrm{~min}$ per patch. The fabricated microneedles were very small with a uniform shape, 
where the tip radius was less than $3.5 \mu \mathrm{m}$ and $400-1000 \mu \mathrm{m}$ in height. Lu et al. (2015) fabricated poly(propylene fumarate)-based microneedles for anticancer drugs, using microstereolithography to treat skin carcinoma. In their studies, poly(propylene fumarate) was mixed with diethyl fumarate to adjust viscosity and improve the mechanical strength. This microneedle system achieved the controlled release of dacarbazine, an anticancer drug for 5 weeks. The drug release rate could be controlled by altering the drug loading and the molecular weight of the polymer monomer (Lu et al. 2015). This study suggests that microstereolithography could be a valuable technique to fabricate drug release devices requiring high structural stabilities.

Although microneedles do not necessarily require biodegradability because they are attached outside the body, needle-shaped microstructures need to penetrate the skin without irritation and, thus, biodegradable scaffolds should be preferable for safety purposes. Recently, Luzuriaga et al. (2018) developed biodegradable microneedles by a new microfabrication technique, using an FDM 3D printer with improved resolution, demonstrating that the printing parameters could be tuned to create microneedles of various shapes, lengths, and array densities, without a master template. The study also showed the degradability of polylactic acid (a renewable, biodegradable, and thermoplastic polymer) in the skin, leading to the drug release (Luzuriaga et al. 2018). Pere et al. (2018) also fabricated biocompatible polymeric microneedle patches for insulin skin delivery. Using the SLA method, a biocompatible resin was photopolymerized to make microneedles of cone and pyramid geometries, followed by IJ print coating of insulin formulations. The developed microneedles, with excellent mechanical strength and piercing capacity, released insulin rapidly within 30 min regardless of the microneedle shapes. Lim et al. (2017) reported a microneedle splint fabricated by 3DP, as a new approach for personalized medicine to treat trigger finger. They prepared the dual function microneedle array on personalized curved surfaces for both effective drug delivery and splinting of affected trigger finger. This microneedle splint showed biocompatibility with human dermal cell lines and significantly enhanced the skin permeation of the loaded-drug (diclofenac) compared to intact skin (Lim et al. 2017).

\section{Others}

3DP technology allows the manufacturing of transdermal delivery systems having highly complex structures and offers some advantages compared to the traditional patch. Recent approaches to combine 3D scanning technology and 3DP methods has facilitated the manufacture of more efficient and personalized patches for drugs (Goyanes et al. 2016b). The 3D scanning system allows acquiring appropriate information about personal skin features, leading to the fabrication of tailored patches by $3 \mathrm{DP}$ techniques. This method is not only limited to patches but also can be used for other attached types of pharmaceutical dosage forms including buccal tablets, thereby enhancing patient compliance.

Specific tissue or cell targeting is important in cancer chemotherapy, and many studies about tumor cell targeting are often focused on using nanoparticles (NPs). Highresolution 3DP technology may be applicable to fabricate NPs with controlled sizes, as delivery systems of anticancer drugs. However, nanoscale fabrication using 3DP has limits due to the lack of materials suitable for nanoscale 3DP. The potential application of 3DP technology in fabricating 3D tissue models for pharmaceutical studies was extensively reviewed by Peng et al. (2017). Compared to traditional twodimensional (2D) models, 3D tissue models provide better in vitro/in vivo correlation at the drug screening stage since they mimic the spatial and chemical attributes of native tissues. Accordingly, recent advances in 3DP technology have accelerated the fabrication of biomimetic constructs applicable in different stages of new drug discovery and development, potentially to replace preclinical animal models. The potential application of 3DP technology in the fabrication of DNA biosensors with superior selectivity against a noncomplementary DNA target has also been reported (Loo et al. 2017).

\section{Conclusion}

Various 3DP methods have been developed and categorized into subgroups, by its working principles. 3DP technology makes it possible to fabricate highly sophisticated and complex dosage forms of drugs and has enhanced the freedom to control the shape and microstructures of dosage forms. Furthermore, 3DP is an innovative and highly promising way for on-demand manufacturing and dosage form personalization, which may improve patient compliance and drug effectiveness, reduce the side effects, resolve the stability issues of drugs with limited shelf-life, and, eventually, lead to the patient-specific health care with on-demand tailored medications. However, in spite of many potential medical and economic benefits, there are also some technical challenges restricting the wide applications of 3DP technology to product commercialization, including the limited choice of biocompatible materials available for 3DP printers, pharmaco-technical issues of current 3DP methods affecting the stability of starting materials, the capacity and reproducibility of processes, and the quality of final products. The regulatory modifications and considerations may also need to be defined for the approval of pharmaceutical products made by 3DP methods. As continuous innovation and refinement in 3DP methods overcome many technical and 
regulatory challenges, rapidly evolving 3DP technology will be more widely applicable to various drug delivery systems and accelerate the clinical practice of more patient-friendly personalized dosage forms in the future.

Acknowledgements This work was supported by the National Research Foundation of Korea (NRF) grant funded by the Korea government (MSIT) (no. 2016R1A2B2010097) and by a grant (16173MFDS542) from Ministry of Food and Drug Safety.

\section{Compliance with ethical standards}

Conflict of interest All authors declare that they have no conflict of interest.

Research involving human participants and animals This article does not contain any studies with human and animal subjects performed by any of the authors.

Open Access This article is distributed under the terms of the Creative Commons Attribution 4.0 International License (http://creativeco mmons.org/licenses/by/4.0/), which permits unrestricted use, distribution, and reproduction in any medium, provided you give appropriate credit to the original author(s) and the source, provide a link to the Creative Commons license, and indicate if changes were made.

\section{References}

Ahlfeld T, Akkineni AR, Förster Y, Köhler T, Knaack S, Gelinsky M, Lode A (2017) Design and fabrication of complex scaffolds for bone defect healing: combined 3D plotting of a calcium phosphate cement and a growth factor-loaded hydrogel. Ann Biomed Eng 45:224-236

Alhijjaj M, Belton P, Qi S (2016) An investigation into the use of polymer blends to improve the printability of and regulate drug release from pharmaceutical solid dispersions prepared via fused deposition modeling (FDM) 3D printing. Eur J Pharm Biopharm 108:111-125

Alhnan MA, Okwuosa TC, Sadia M, Wan K-W, Waqar A, Basel A (2016) Emergence of 3D printed dosage forms: opportunities and challenges. Pharm Res 33:1817-1832

Beck RCR, Chaves PS, Goyanes A, Vukosavljevic B, Buanz A, Windbergs M, Basit AW, Gaisford S (2017) 3D printed tablets loaded with polymeric nanocapsules: an innovative approach to produce customized drug delivery systems. Int J Pharm 528:268-279

Bezuidenhout MB, Dimitrov DM, van Staden AD, Oosthuizen GA, Dicks LMT (2015) Titanium-based hip stems with drug delivery functionality through additive manufacturing. BioMed Res Int 2015:11

Bikas H, Stavropoulos P, Chryssolouris G (2016) Additive manufacturing methods and modelling approaches: a critical review. Int J Adv Manuf Technol 83:389-405

Chia HN, Wu BM (2015) Recent advances in 3D printing of biomaterials. J Biol Eng 9:4

Clark EA, Alexander MR, Irvine DJ, Roberts CJ, Wallace MJ, Sharpe S, Yoo J, Hague RJM, Tuck CJ, Wildman RD (2017) 3D printing of tablets using inkjet with UV photoinitiation. Int J Pharm 529:523-530

Dimitrov D, Schreve K, de Beer N (2006) Advances in three dimensional printing - state of the art and future perspectives. Rapid Prototyp J 12:136-147
Gans-de B, Duineveld P, Schubert U (2004) Inkjet printing of polymers: state of the art and future developments. Adv Mater $16: 203-213$

Genina N, Holländer J, Jukarainen H, Mäkilä E, Salonen J, Sandler N (2016) Ethylene vinyl acetate (EVA) as a new drug carrier for 3D printed medical drug delivery devices. Eur J Pharm Sci 90:53-63

Goole J, Amighi K (2016) 3D printing in pharmaceutics: a new tool for designing customized drug delivery systems. Int J Pharm 499:376-394

Goyanes A, Buanz ABM, Basit AW, Gaisford S (2014) Fused-filament 3D printing (3DP) for fabrication of tablets. Int J Pharm 476:88-92

Goyanes A, Buanz AB, Hatton GB, Gaisford S, Basit AW (2015a) 3D printing of modified-release aminosalicylate (4-ASA and 5-ASA) tablets. Eur J Pharm Biopharm 89:157-162

Goyanes A, Wang J, Buanz A, Martínez-Pacheco R, Telford R, Gaisford S, Basit AW (2015b) 3D printing of medicines: engineering novel oral devices with unique design and drug release characteristics. Mol Pharm 12:4077-4084

Goyanes A, Chang H, Sedough D, Hatton GB, Wang J, Buanz A, Gaisford S, Basit AW (2015c) Fabrication of controlled-release budesonide tablets via desktop (FDM) 3D printing. Int J Pharm 496:414-420

Goyanes A, Robles Martinez P, Buanz A, Basit AW, Gaisford S (2015d) Effect of geometry on drug release from $3 \mathrm{D}$ printed tablets. Int $\mathrm{J}$ Pharm 494:657-663

Goyanes A, Kobayashi M, Martinez-Pacheco R, Gaisford S, Basit AW (2016a) Fused-filament 3D printing of drug products: microstructure analysis and drug release characteristics of PVA-based caplets. Int J Pharm 514:290-295

Goyanes A, Det-Amornrat U, Wang J, Basit AW, Gaisford S (2016b) $3 \mathrm{D}$ scanning and $3 \mathrm{D}$ printing as innovative technologies for fabricating personalized topical drug delivery systems. J Control Release 234:41-48

Goyanes A, Fina F, Martorana A, Sedough D, Gaisford S, Basit AW (2017) Development of modified release 3D printed tablets (printlets) with pharmaceutical excipients using additive manufacturing. Int J Pharm 527:21-30

Gross BC, Erkal JL, Lockwood SY, Chen C, Spence DM (2014) Evaluation of 3D printing and its potential impact on biotechnology and the chemical sciences. Anal Chem 86:3240-3253

Huang W, Zheng Q, Sun W, Xu H, Yang X (2007) Levofloxacin implants with predefined microstructure fabricated by threedimensional printing technique. Int J Pharm 339:33-38

Jacob J, Coyle N, West TG, Monkhouse DC, Surprenant HL, Jain NB (2014) Rapid disperse dosage form containing levetiracetam. WO2014144512A1

Johnson AR, Caudill CL, Tumbleston JR, Bloomquist CJ, Moga KA, Ermoshkin A, Shirvanyants D, Mecham SJ, Luft JC, DeSimone JM (2016) Single-step fabrication of computationally designed microneedles by continuous liquid interface production. PLoS ONE 11:e0162518

Katstra WE, Palazzolo RD, Rowe CW, Giritlioglu B, Teung P, Cima MJ (2000) Oral dosage forms fabricated by three dimensional printing. J Control Release 66:1-9

Khaled SA, Burley JC, Alexander MR, Roberts CJ (2014) Desktop 3D printing of controlled release pharmaceutical bilayer tablets. Int J Pharm 461:105-111

Khaled SA, Burley JC, Alexander MR, Yang J, Roberts CJ (2015a) 3D printing of five-in-one dose combination polypill with defined immediate and sustained release profiles. J Control Release 217:308-314

Khaled SA, Burley JC, Alexander MR, Yang J, Roberts CJ (2015b) 3D printing of tablets containing multiple drugs with defined release profiles. Int J Pharm 494:643-650 
Khaled SA, Alexander MR, Wildman RD, Wallace MJ, Sharpe S, Yoo J, Roberts CJ (2018) 3D extrusion printing of high drug loading immediate release paracetamol tablets. Int J Pharm 538:223-230

Kim K, Han S, Yoon J, Kwon S, Park H-K, Park W (2016) Lithographic resolution enhancement of a maskless lithography system based on a wobulation technique for flow lithography. Appl Phys Lett 109:234101

Konta AA, Garcia-Pina M, Serrano DR (2017) Personalised 3D printed medicines: which techniques and polymers are more successful? Bioengineering (Basel) 4:79-94

Kyobula M, Adedeji A, Alexander MR, Saleh E, Wildman R, Ashcroft I, Gellert PR, Roberts CJ (2017) 3D inkjet printing of tablets exploiting bespoke complex geometries for controlled and tuneable drug release. J Control Release 261:207-215

Li Q, Guan X, Cui M, Zhu Z, Chen K, Wen H, Jia D, Hou J, Xu W, Yang X, Pan W (2018) Preparation and investigation of novel gastro-floating tablets with $3 \mathrm{D}$ extrusion-based printing. Int $\mathrm{J}$ Pharm 535:325-332

Lim SH, Ng JY, Kang L (2017) Three-dimensional printing of a microneedle array on personalized curved surfaces for dualpronged treatment of trigger finger. Biofabrication 9:015010

Loo AH, Chua CK, Pumera M (2017) DNA biosensing with 3D printing technology. Analyst 142:279-283

Lu Y, Satya Nymisha M, Douglas CC, Sofia C, Kush NS, Yang HY, Ryan BW, Jae-Won C (2015) Microstereolithography and characterization of poly(propylene fumarate)-based drug-loaded microneedle arrays. Biofabrication 7:045001

Luzuriaga MA, Berry DR, Reagan JC, Smaldone RA, Gassensmith JJ (2018) Biodegradable 3D printed polymer microneedles for transdermal drug delivery. Lab Chip 18:1223-1230

Maulvi F, Shah MJ, Solanki BS, Patel AS, Soni TG (2017) Application of 3D printing technology in the development of novel drug delivery systems. Int J Drug Dev Res 9:44-49

Melchels FP, Feijen J, Grijpma DW (2010) A review on stereolithography and its applications in biomedical engineering. Biomaterials 31:6121-6130

Murphy SV, Atala A (2014) 3D bioprinting of tissues and organs. Nat Biotechnol 32:773-785

Norman J, Madurawe RD, Moore CM, Khan MA, Khairuzzaman A (2017) A new chapter in pharmaceutical manufacturing: 3D-printed drug products. Adv Drug Deliv Rev 108:39-50

Ochoa M, Zhou J, Rahimi R, Badwaik V, Thompson D, Ziaie B (2015) Rapid 3D-print-and-shrink fabrication of biodegradable microneedles with complex geometries. In: 2015 Transducers-2015 18th international conference on solid-state sensors, actuators and microsystems (TRANSDUCERS), pp 1251-1254

Okwuosa TC, Stefaniak D, Arafat B, Isreb A, Wan KW, Alhnan MA (2016) A lower temperature FDM 3D printing for the manufacture of patient-specific immediate release tablets. Pharm Res 33:2704-2712

Peng W, Datta P, Ayan B, Ozbolat V, Sosnoski D, Ozbolat IT (2017) 3D bioprinting for drug discovery and development in pharmaceutics. Acta Biomater 57:26-46

Pere CPP, Economidou SN, Lall G, Ziraud C, Boateng JS, Alexander BD, Lamprou DA, Douroumis D (2018) 3D printed microneedles for insulin skin delivery. Int J Pharm 544:425-432

Pietrzak K, Isreb A, Alhnan MA (2015) A flexible-dose dispenser for immediate and extended release 3D printed tablets. Eur J Pharm Biopharm 96:380-387

Rowe CW, Katstra WE, Palazzolo RD, Giritlioglu B, Teung P, Cima MJ (2000) Multimechanism oral dosage forms fabricated by three dimensional printing. J Control Release 66:11-17
Sadia M, Sosnicka A, Arafat B, Isreb A, Ahmed W, Kelarakis A, Alhnan MA (2016) Adaptation of pharmaceutical excipients to FDM 3D printing for the fabrication of patient-tailored immediate release tablets. Int J Pharm 513:659-668

Sandler N, Salmela I, Fallarero A, Rosling A, Khajeheian M, Kolakovic R, Genina N, Nyman J, Vuorela P (2014) Towards fabrication of $3 \mathrm{D}$ printed medical devices to prevent biofilm formation. Int $\mathrm{J}$ Pharm 459:62-64

Skowyra J, Pietrzak K, Alhnan MA (2015) Fabrication of extendedrelease patient-tailored prednisolone tablets via fused deposition modelling (FDM) 3D printing. Eur J Pharm Sci 68:11-17

Sumerel J, Lewis J, Doraiswamy A, Deravi LF, Sewell SL, Gerdon AE, Wright DW, Narayan RJ (2006) Piezoelectric ink jet processing of materials for medical and biological applications. Biotechnol J Healthcare Nutr Technol 1:976-987

Sun Y, Soh S (2015) Printing tablets with fully customizable release profiles for personalized medicine. Adv Mater 27:7847-7853

Tirella A, Vozzi F, Vozzi G, Ahluwalia A (2011) PAM2 (piston assisted microsyringe): a new rapid prototyping technique for biofabrication of cell incorporated scaffolds. Tissue Eng Part C Methods 17:229-237

Vaezi M, Seitz H, Yang S (2013) A review on 3D micro-additive manufacturing technologies. Int J Adv Manuf Technol 67:1721-1754

Verstraete G, Samaro A, Grymonpré W, Vanhoorne V, Van Snick B, Boone MN, Hellemans T, Van Hoorebeke L, Remon JP, Vervaet C (2018) 3D printing of high drug loaded dosage forms using thermoplastic polyurethanes. Int J Pharm 536:318-325

Vozzi G, Flaim C, Ahluwalia A, Bhatia S (2003) Fabrication of PLGA scaffolds using soft lithography and microsyringe deposition. Biomaterials 24:2533-2540

Wang C-C, Tejwani MR, Roach WJ, Kay JL, Yoo J, Surprenant HL, Monkhouse DC, Pryor TJ (2006) Development of near zero-order release dosage forms using three-dimensional printing (3-DPTM) technology. Drug Dev Ind Pharm 32:367-376

Wang J, Goyanes A, Gaisford S, Basit AW (2016) Stereolithographic (SLA) 3D printing of oral modified-release dosage forms. Int $\mathbf{J}$ Pharm 503:207-212

Wu W, Zheng Q, Guo X, Sun J, Liu Y (2009) A programmed release multi-drug implant fabricated by three-dimensional printing technology for bone tuberculosis therapy. Biomed Mater 4:065005

Wu W, Ye C, Zheng Q, Wu G, Cheng Z (2016) A therapeutic delivery system for chronic osteomyelitis via a multi-drug implant based on three-dimensional printing technology. J Biomater Appl 31:250-260

Yi HG, Choi YJ, Kang KS, Hong JM, Pati RG, Park MN, Shim IK, Lee CM, Kim SC, Cho DW (2016) A 3D-printed local drug delivery patch for pancreatic cancer growth suppression. J Control Release 238:231-241

Yu DG, Yang XL, Huang WD, Liu J, Wang YG, Xu H (2007) Tablets with material gradients fabricated by three-dimensional printing. J Pharm Sci 96:2446-2456

Yu D-G, Branford-White C, Ma Z-H, Zhu L-M, Li X-Y, Yang X-L (2009) Novel drug delivery devices for providing linear release profiles fabricated by 3DP. Int J Pharm 370:160-166

Zhang J, Feng X, Patil H, Tiwari RV, Repka MA (2017) Coupling $3 \mathrm{D}$ printing with hot-melt extrusion to produce controlled-release tablets. Int J Pharm 519:186-197 